Published online 2017 April 13.

Abstract

\title{
Imaging of Anterior Skull Base Pathologies
}

Leila Aghaghazvini ${ }^{1}{ }^{*}$

${ }^{1}$ Tehran University of Medical Sciences, Shariati Hospital, Tehran, Iran

"Corresponding author: Leila Aghaghazvini, MD, Tehran University of Medical Sciences, Shariati Hospital, Tehran, Iran. E-mail: aghaghazvini.leila@gmail.com

Received 2016 December 21; Accepted 2017 February 08.

\begin{abstract}
Several lesions (Inflammatory, neoplastic and congenital) can involve in the skull base. The role of imaging is to detect the location and extension of lesion, determine mass and mass like lesions, differentiate benign from malignant tumors, detect invasion to adjacent structures and make decision in surgical planning. Skull base divides to anterior, middle and posterior cranial fossa and some pathologies are common in all spaces and a few are only seen in special sites. In this presentation we plan to explain the lesions in anterior skull base.
\end{abstract}

This is an abstract presented in the 33rd Iranian congress of radiology (ICR) and the 15th congress of Iranian radiographic science association (IRSA). 